\title{
Vision-related quality of life in children with glaucoma
}

\author{
Benjamin L. Freedman ${ }^{a}$, Sarah K. Jones, BS ${ }^{b}$, Alice Lin, MD ${ }^{b}$, Sandra S. Stinnett, DrPH ${ }^{b}$, \\ and Kelly W. Muir, MD, MHSc ${ }^{\text {b,c }}$ \\ aUniversity of Pennsylvania, Philadelphia \\ bDuke Eye Center, Durham, North Carolina \\ cDurham VA Medical Center, Durham, North Carolina
}

\begin{abstract}
Although reporting vision-related quality of life (VRQoL) outcomes has become increasingly common in adult glaucoma studies, little is known about the influence of disease severity, medication burden, and surgical experience on the quality of life of children with glaucoma. We tested the feasibility of administering a self-reported VRQoL instrument and describe the VRQoL in children with glaucoma. Better VRQoL was correlated with higher visual acuity in the betterseeing eye but not the number of surgeries the child had undergone or the number of prescribed eyedrops.
\end{abstract}

\section{Methods}

With approval of the Duke University Medical Center Institutional Review Board and in compliance with the US Health Information Portability and Accountability Act of 1996, consecutive patients aged 5-17 years were approached regarding study participation. A sample size of 50 subjects was chosen empirically. Inclusion criteria were age $<18$ years, glaucoma diagnosis and $\geq 1$ prescribed glaucoma medication. Patients who had undergone ophthalmic surgery within the last month and those expected to undergo ophthalmic surgery within the following month were excluded. This cross-sectional study was part of a previously reported ${ }^{1}$ prospective investigation of medication adherence.

At the baseline visit, subjects were given the Impact of Vision Impairment on Children (IVIC), a 24-item VRQoL instrument developed from thematic analysis of focus group discussion of children with visual impairment. ${ }^{2}$ The IVI-C has excellent psychometric properties and has been validated in children with and without visual impairment. ${ }^{3}$ It is scored from 0 to 96, with lower scores representing better VRQoL (see e-Supplement 1, available at jaapos.org, for an excerpt).

The chart was abstracted for demographic information as well as glaucoma diagnosis and treatment history, including previous glaucoma surgeries, prescribed glaucoma medications, and best-corrected visual acuity. Surgeries were defined as incisional ophthalmic surgery (excluding examination under anesthesia without surgery). Snellen visual acuities were converted to $\log$ MAR for analysis. Means for continuous variables between groups were compared with the $t$ test or analysis of variance; the relationship between continuous

Correspondence: Kelly W. Muir, MD, MHS, Duke University Medical Center, Box 3802, Durham, NC 27710 (kelly.muir@duke.edu). Publisher's Disclaimer: This is a PDF file of an unedited manuscript that has been accepted for publication. As a service to our customers we are providing this early version of the manuscript. The manuscript will undergo copyediting, typesetting, and review of the resulting proof before it is published in its final citable form. Please note that during the production process errorsmaybe discovered which could affect the content, and all legal disclaimers that apply to the journal pertain. 
variables was assessed with linear regression, with $P<0.05$ considered statistically significant.

\section{Results}

Of the 50 subjects approached to participate, 43 (age range, 5-17 years) were able to complete the self-reported IVI-C instrument (Table 1). Scores ranged from 0 to 56 (mean, $24 \pm 13$ [SD]; median, 24; Figure 1). The score was not related to sex $(P=0.229)$, age $(P=$ $0.054)$, race $(P=0.520)$, bilaterality or unilaterality of disease $(P=0.253)$, the number of eye surgeries $(P=0.638)$, the number of glaucoma medications prescribed $(P=0.943)$, or the frequency of prescribed dosing $(0.932)$.

Visual acuity in the better-seeing eye ranged from 20/15 to 1/400 (median, 20/25). In linear regression analysis, sharper visual acuity in the better-seeing eye was associated with better VRQoL $(P=0.031)$. Considering that subjects with unilateral glaucoma are likely to have normal ocular function in the unaffected eye, we repeated the linear regression analysis, including only subjects with bilateral glaucoma $(\mathrm{n}=32)$, and sharper visual acuity was again associated with better VRQoL $(P=0.013)$. Additionally, because the range of visual acuity for the better-seeing eye was skewed toward good vision, we further investigated the relationship by dichotomizing the subjects with bilateral disease into two groups: children with visual acuity of $\geq 20 / 40$ in the better-seeing eye $(n=14)$ and children with visual acuity $<20 / 40(n=18)$. This cut-off point was chosen because it is a common value used to determine whether or not a person is granted an unrestricted driver's license. The median IVI-C score for the group of children with greater visual acuity in the better-seeing eye (median IVI-C, 17) was significantly lower, indicating better quality of life, than the mean IVI-C score for the children with worse visual acuity (median IVI-C, Bo27; $P=0.023$ [Mann-Whitney U test]; Figure 2). Given the borderline significance of age with VRQoL in univariable analyses $(P=0.054)$, age and visual acuity in the better-seeing eye were considered together as potential explanatory variables for VRQoL in a multiple regression model. Sharper visual acuity was associated with better VRQoL $(P=0.031)$ and the association between age and VRQoL was of borderline significance $(P=0.052)$.

The IVI-C instrument was validated only in children 8-18 years of age and our study included some younger subjects. We therefore performed all analyses on both the whole group and on the subset of subjects $8-18$ years of age with equivalent results (data not shown). The number of participants $<8$ years of age was inadequate to allow for statistical analysis.

\section{Discussion}

Various instruments have been developed to assess VRQoL in children, ${ }^{4-6}$ but most depend on proxy report rather than child response. ${ }^{4}$ The IVI-C was developed with focus groups of children with visual impairment, an important developmental step toward creating an instrument that addresses the unique concerns of children. ${ }^{2}$

In this cohort, sharper visual acuity in the better-seeing eye was associated with better VRQoL. Similarly, in studies of children with low birth weight, better visual acuity was associated with superior health-related quality of life. ${ }^{7}$ The relationship between visual acuity in the better-seeing eye and better VRQoL noted in the present study population, despite good median visual acuity, is consistent with the literature regarding adults with glaucoma, in whom loss of visual acuity seems to be more important than loss of visual field in determining VRQoL. ${ }^{8}$ Reliable visual field testing is not always possible in young children and visual field testing was accordingly not included in this study. In a study of 
children undergoing surgery for primary congenital glaucoma in China, investigators also found a positive association between worse postoperative visual acuity and worse quality of life. ${ }^{9}$

Children may face unique challenges related to their glaucoma. We hypothesized that children prescribed a greater number of glaucoma medications would score worse on the VRQoL instrument than children prescribed fewer medications because of school and social activities. We also postulated that children having undergone multiple surgeries would report worse VRQoL due to social stigma. However, in both the total sample with all IVI-C items and in subanalyses of all 43 subjects using only the subgroup of questions related to school and social activities (data not shown), neither the number of prescribed medications nor the number of glaucoma surgeries was associated with VRQoL. However, conducting the analysis on a smaller set of item responses could increase variability and decrease the power to detect significant differences.

This study is limited by its relatively small sample size and is subject to selection bias. Although we made every attempt to approach consecutive patients, as no potential subjects refused participation, we may have inadvertently approached more willing participants, who may have been happier with their care and with greater perceived quality of life than children who were not approached. Although previous work shows that the IVI-C is well suited to the Rasch model, ${ }^{3}$ we did not utilize Rasch analysis in this pilot study. Larger studies of children with a variety of visual disorders would benefit from Rasch linear scaling to compare VRQoL among groups.

To our knowledge, this is the first self-reported assessment of VRQoL in children with different types of glaucoma. As novel therapeutic options for children with glaucoma are developed, it will be important to consider quality-of-life as a crucial outcome variable in evaluating treatment success.

\section{Literature Search}

MEDLINE was searched via PubMed on September 9, 2013, without date restriction, for English-language results using the following keywords: childhood glaucoma, quality-of-life.

\section{Supplementary Material}

Refer to Web version on PubMed Central for supplementary material.

\section{Acknowledgments}

Supported by 5K12 EY016333-05. Dr. Muir receives salary support from the VA Health Services Research and Development, VA CDA 10-019-2. The funding organizations had no role in the design or conduct of this research.

\section{References}

1. Freedman RB, Jones SK, Lin A, Robin AL, Muir KW. Influence of parental health literacy and dosing responsibility on pediatric glaucoma medication adherence. Arch Ophthalmol. 2012; 130:306-11. [PubMed: 22411659]

2. Cochrane G, Lamoureux E, Keeffe J. Defining the content for a new quality of life questionnaire for students with low vision (the Impact of Vision Impairment on Children: IVI_C). Ophthalmic Epidemiol. 2008; 15:114-20. [PubMed: 18432495]

3. Cochrane GM, Marella M, Keeffe JE, Lamoureux EL. The Impact of Vision Impairment for Children (IVI_C): validation of a vision-specific pediatric quality-of-life questionnaire using Rasch analysis. Invest Ophthalmol Vis Sci. 52:1632-40. [PubMed: 21178144] 
4. Felius J, Stager DR Sr, Berry PM, et al. Development of an instrument to assess vision-related quality of life in young children. Am J Ophthalmol. 2004; 138:362-72. [PubMed: 15364217]

5. Gothwal VK, Lovie-Kitchin JE, Nutheti R. The development of the LV Prasad-Functional Vision Questionnaire: a measure of functional vision performance of visually impaired children. Invest Ophthalmol Vis Sci. 2003; 44:4131-9. [PubMed: 12939337]

6. Khadka J, Ryan B, Margrain TH, Court H, Woodhouse JM. Development of the 25-item Cardiff Visual Ability Questionnaire for Children (CVAQC). Br J Ophthalmol. 2010; 94:730-35. [PubMed: 20508047]

7. Quinn GE, Dobson V, Saigal S, et al. CRYO-ROP Cooperative Group. Health-related quality of life at age 10 years in very low-birth-weight children with and without threshold retinopathy of prematurity. Arch Ophthalmol. 2004; 122:1659-66. [PubMed: 15534127]

8. Parrish RK 2nd, Gedde SJ, Scott IU, et al. Visual function and quality of life among patients with glaucoma. Arch Ophthalmol. 1997; 115:1447-55. [PubMed: 9366678]

9. Zhang XL, Du SL, Ge J, et al. Quality of life in patients with primary congenital glaucoma following antiglaucoma surgical management. Zhonghua Yan Ke Za Zhi. 2009; 45:514-21. [PubMed: 19957674] 


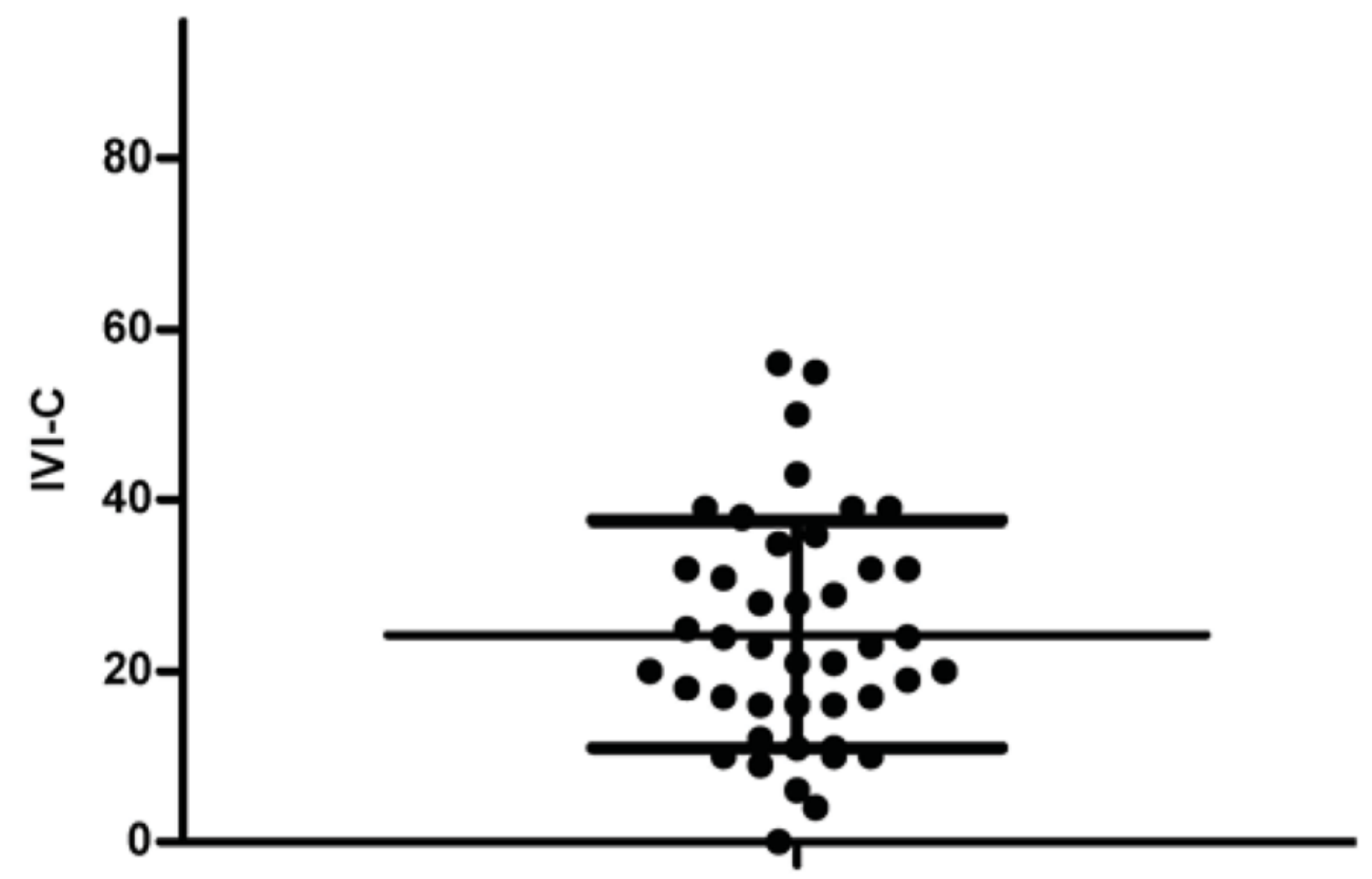

\section{All subjects completing IVI-C $(n=43)$}

FIG 1.

Distribution of vision-related quality-of-life scores for children with glaucoma. Dots represent scores of each of the 43 children with glaucoma who took the Inventory of Visual Impairment in Children (IVI-C), scaled 0-96, with higher scores for worse vision-related quality of life. The bar represents the mean (24); the whiskers, the standard deviation (13). 


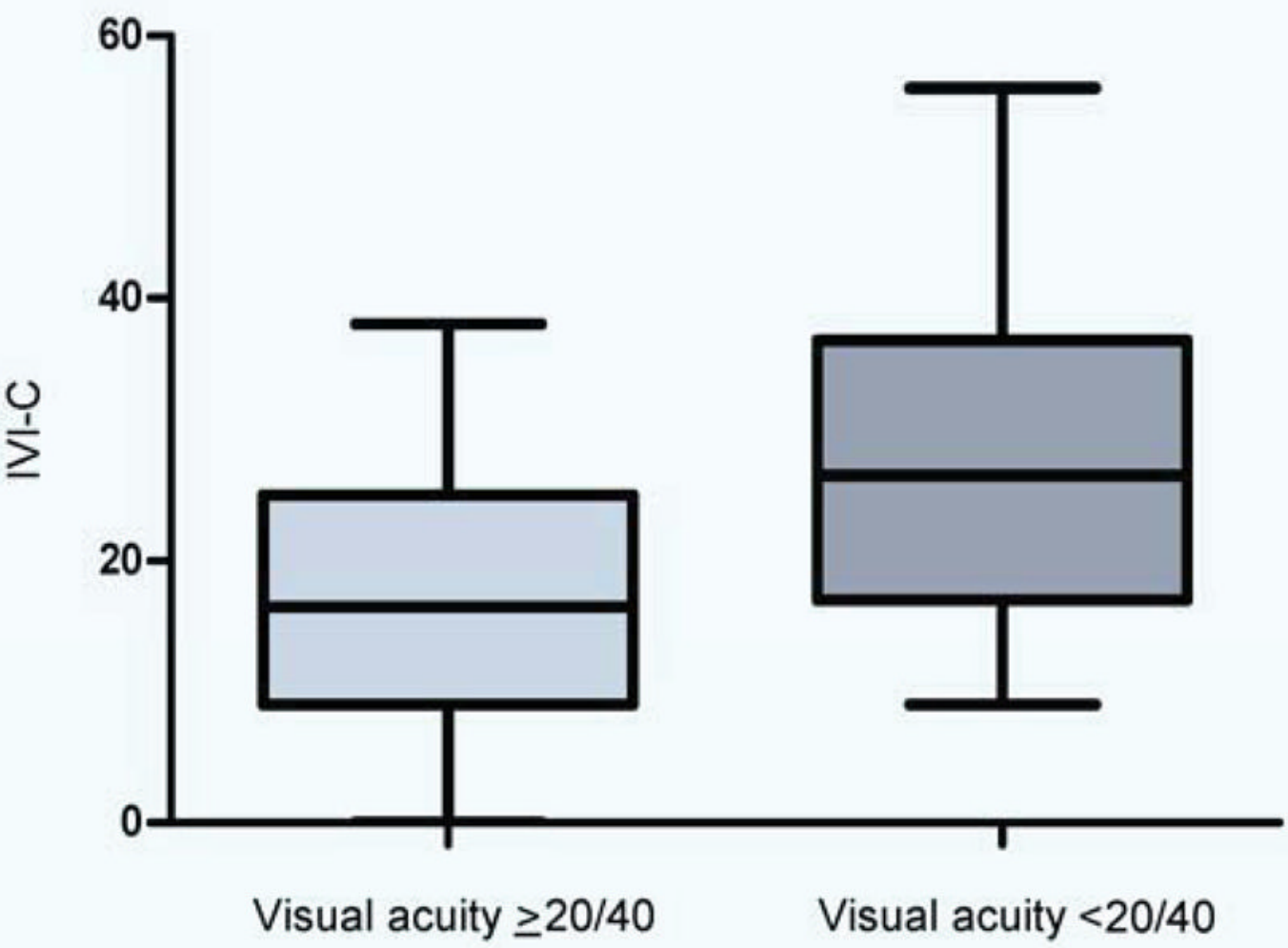

FIG 2.

Vision-related quality of life according to visual acuity in the better-seeing eye. Box-andwhiskers plots describe the scores on the IVI-C survey for the 14 children with bilateral glaucoma and visual acuity in the better-seeing eye of $\geq 20 / 40$ on the left, and scores for the 18 children with bilateral glaucoma and visual acuity in the better-seeing eye of $<20 / 40$ on the right. The median IVI-C scores for children with visual acuity in the better-seeing eye $20 / 40$ were significantly lower than the scores of the children with visual acuity in the better-seeing eye $<20 / 40$. 
Table 1

Characteristics of study subjects completing the Impact of Vision Impairment on Children Instrument

\begin{tabular}{ll}
\hline Subject characteristics $(\mathbf{n}=\mathbf{4 3})$ & Number of subjects \\
\hline Sex (male/female) & $24 / 19$ \\
Race & 23 \\
$\quad$ White & 13 \\
African American & 7 \\
Other/unknown & \\
Visual acuity in the better-seeing eye & $10 / 14$ \\
$\quad \geq 20 / 40$, unilateral/bilateral glaucoma & $1 / 18$ \\
$\quad<20 / 40$, unilateral/bilateral glaucoma & \\
Glaucoma diagnosis & 13 \\
Congenital glaucoma & 16 \\
Aphakic glaucoma after pediatric cataract removal & 14 \\
Other & $11 / 32$ \\
Laterality, unilateral/bilateral & Value \\
Number glaucoma surgeries, mean \pm SD (median) & $5 \pm 3(5)$ \\
Number glaucoma medications, mean \pm SD (median) & $2 \pm 1(2)$ \\
Age in years, mean \pm SD (median) & $11 \pm 4(10)$ \\
\hline
\end{tabular}

